\title{
Cyclic animation using Partial Differential Equations
}

\author{
G. González Castro • M. Athanasopoulos · H. Ugail
}

Received: October 29, 2009/ Accepted: date

\begin{abstract}
This work presents an efficient and fast method for achieving cyclic animation using Partial Differential Equations (PDEs). The boundary-value nature associated with elliptic PDEs offers a fast analytic solution technique for setting up a framework for this type of animation. The surface of a given character is thus created from a set of pre-determined curves, which are used as boundary conditions so that a number of PDEs can be solved. Two different approaches to cyclic animation are presented here. The first consists of using attaching the set of curves to a skeletal system holding the animation for cyclic motions linked to a set mathematical expressions, the second one exploits the spine associated with the analytic solution of the PDE as a driving mechanism to achieve cyclic animation, which is also manipulated mathematically. The first of these approaches is implemented within a framework related to cyclic motions inherent to human-like characters, whereas the spine-based approach is focused on modelling the undulatory movement observed in fish when swimming. The proposed method is fast and accurate. Additionally, the animation can be either used in the PDE-based surface representation of the model or transferred to the original mesh model by means of a point to point map. Thus, the user is offered with the choice of using either of these two animation representations of the same object, the selection depends on the computing resources such as storage and memory capacity associated with each particular application.
\end{abstract}

\section{F. Author}

first address

Tel.: + 123-45-678910

Fax: +123-45-678910

E-mail: fauthor@example.com

S. Author

second address
Keywords First keyword · Second keyword · More

\section{Introduction}

Cyclic animation is a loopable animation of a character repeating an action that can be used to move a character along a path easily and realistically. In other words, A cyclic animation involves the construction of a sequence of frames that can be used for repetitive motions [26]. Thus, the animator can save time by animating a single cycle, rather than manually animating every footstep. This technique is ideal for repetitive motions, such as a character walking, running, dancing or swimming. For instance, a walking cycle is composed of taking a step forward with one foot and then another step with the other foot, creating a loop such that when the sequence is repeated, the resulting motion is seamless.

Generally, this type of animations is obtained by adjusting a skeleton system to a given model which will be responsible for storing and manipulating the frame sequence. This technique, often referred as rigging, uses several "bones" or joints connected to each other to control the overall movement of the skeleton. The motion of this skeleton is generally created manually by positioning the handles controlling the joints to different key poses. Afterwards, several interpolation techniques can be applied to generate the in-between positions. For further information on these techniques, the reader is referred to [18] where such techniques are explained in detail. It is worth mentioning that the movement of the joints can also be controlled by applying a suitable set of mathematical expressions. These mathematical expressions are chosen so that the generated animation simulates realistic movement. The work presented here 
makes use of mathematical expressions to control the attributes of the objects.

After assigning and designing the motion over the skeletal system, this structure is attached to a single mesh object, which is deformed according to the motion embedded in the skeleton. This technique is called skinning and, given that this work exploits the use of PDEs as a surface generation technique, this work uses PDE surfaces attached to the skeletal system. In the interest of illustrating technique proposed here for skeletal cyclic animation, different movements associated with humans are employed

Additionally, the use of PDEs for cyclic animation can be exploited further by taking advantage of the mathematical properties associated with PDE surfaces rising from elliptic PDEs: cyclic animation can benefit from the existence of an analytic expression describing the spine of a given object. This can be carried out by manipulating the spine as if it was the skeletal system driving the animation. Spine based cyclic animation is studied through the simulation of fish locomotion.

Finally, a point to point map has been developed to transfer the animation to different objects than can be either PDE surface representations or complex mesh models and thus enabling the possibility to have multiple human objects sharing the same animation.

\subsection{Related work}

Both, human motion and fish locomotion have been areas attracting the interest of the most diverse disciplines. Efficient simulation of such movements over virtual environments has become increasingly demanding in terms of the amount of resources employed and realism achieved. Given the familiarity we all have with human cyclic motion, no description of such movement is included; however, an outline of the work carried out so far for simulating them is given below.

\subsubsection{Human animation}

The major problem arising from simulating human cyclic motions is that of preserving the realism with which such movements take place. Moreover, faster and more accurate techniques are in constant demand due to the advances in technology. Therefore, extensive work has been undertaken in the area including:

- An automated method for modelling cyclic 3D motion [19]. This work developed an algorithm enforcing smooth transitions between cycles by operating in the Fourier domain. The major contribution of this method A key point in this method consists of its ability to automatically deal with noise and missing data.

- A method for learning and tracking human motion in video [20]. This technique is capable of converting large sets of periodic human motion data automatically into cycles. The learned temporal model provides a prior probability distribution over human motion that can potentially be used for tracking human subjects in complex monocular video sequences and recovering their motion.

- A technique that allows content creators to easily integrate virtual humans into Web3D virtual environments, such as X3D and VRML [15]. This technique allows the user to interactively integrate virtual humans acting as formal instructors in virtual environments for learning or training purposes.

- A three layer hierarchical control system for animating human avatars in $3 \mathrm{D}$ virtual environments [32]. The first layer controls the movement of the avatar's joints, the second defines skills or basic behaviors and the highest layer executes a behaviour-based script language that can be used to describe stories to be performed by the avatars.

It is the understanding of the authors that so far, cyclic human motion has been achieved by using mesh models exclusively. This may potentially give rise to modification of the mesh model in order to correct the shape once it has been animated. Given that it is desirable to have control over the shape of the surface once it has been defined. For that reason, the use of PDE surfaces gives us the advantage to represent a surface analytically, thus making the manipulation of that surface fast and accurate.

\subsubsection{Fish locomotion}

The movement observed by fish when swimming has captured the attention of different disciplines varying from biology and fluid mechanics to areas such as robotics and computer animation. The reasons for which these disciplines are interested are diverse. For instance, biologists are interested in how evolutionary aspects have determined the form of swimming among different types of aquatic animals. Creative disciplines require to simulate as realistically as possible this type of natural phenomena. However, a number of factors affect accuracy of simulation in areas such as computer graphics and computer animation. This movement can be divided into two categories namely the periodic propulsion and transient movements. The first category is thought to be responsible for the displacement, whereas the second one comprises sudden movements generally associated 
with abrupt changes on speed and direction, which generally occur after the sensorial identification of an obstacle and predator or prey accordingly. The full characterization of the undulatory movement observed in most aquatic animals when swimming has been outlined in [16] associating it as faithfully as possible with the concepts related to hydromechanics. This work divides the periodic motion observed in aquatic animals into four categories as follows:

- Anguilliform. This movement is observed in long fish such as eels and tile-fish and distinguishes from the others since the whole body length is used throughout. Additionally, it is considered as the most basic form of undulatory propulsion.

- Subcarangiform. Trout and cod are examples of fish possessing this type of movement. This type of movement is considered as an intermediate mode between the anguilliform and carangiform modes [23]. The undulatory effect is observed in the posterior region with the amplitude increasing towards the tail. In this case the moving region can consist of two thirds of the length of the fish as maximum.

- Carangiform. Among the fish presenting this kind of movement pattern can be found sea-bass, barracuda and gold fish. The main feature characterizing this movement is the fact that the undulatory nature of the movement is visible on the posterior half of the body which may be provoked by the fact that this part of the body is more flexible than the rest.

- Thunniform. Some of the aquatic animals presenting this movement are regarded as the fastest fish. These fish are characterized by a lunate-shape tail fin, which is thought to be mainly responsible for the noticeable increase in speed. The movement of the body is basically restricted to the tail.

Mathematically, the general nature of the undulatory movement has been modelled using a travelling wave advancing in the opposite direction of movement from where the four subcategories can be modelled by adjusting the values of a set of parameters. Additionally, a number of studies have been undertaken in order to determine how fish locomotion is affected by the hydrodynamic effects such as vortex and drag [36]. Some other disciplines rather far away from biology and fluid mechanics, have been interested in the qualitative aspects of fish locomotion. For instance, the design of robots capable of swimming so that these robots can indistinctively merge with some other real fish. Some of the work carried out in computer aided design and simulation include:

- First attempt for achieving realistic model of a fish in computer animation [27]. This work includes as- pects related to physics, movement, perception and behaviour and adopts a spring-mass model so that the undulatory nature of the movement is included.

- Development of virtual reality environments as in [14]

- Development of computer animation techniques [24].

- The study of social behaviour (prey-predator relations) ans collision detection using fuzzy logic as means accelerate the solution of such systems [2].

According to [34], the full simulation of the dynamics of the fish model is determined by sixty-nine ordinary differential equations modelling the appropriate muscle contractions responsible for deforming the mesh model. The system is mainly governed by four springs called motor controllers and account for the amplitude and speed of the undulatory movement. Moreover, as far as we are aware, most of the work carried out so far in fish animation and virtual reality environments involving fish locomotion are based on the spring-mass model as described.

Generally, spring-mass models are solved using numerical techniques such as the semi-implicit Euler method. Such numerical methods present a tendency to be either slow or prone to numerical errors, which may lead to inaccuracies affecting the geometric model.

Thus, our motivation for this work is to develop a simple analytic technique for simulation of fish locomotion, which may prove useful in addressing some of the shortcomings of existing techniques. Hence, a parametric surface representation of a fish and its corresponding manipulation are natural candidates to pursue this direction. Analytic solutions to particular elliptic PDEs may be exploited as a tool for achieving fish locomotion by taking advantage of their mathematical properties.

\subsection{Contribution}

This work makes use of PDE based surfaces in cyclic animation in two different manners. The first uses PDE surfaces as the skin of a skeletal system, which is responsible for driving the animation. The second one exploits the spine as means for driving the animation. These two approaches provide a new perspective to cyclic animation that addresses some of the problems pertaining to already existing techniques. Moreover, given that the analytic solution of the PDE can be use to drive the animation itself, avoids complicationsinherent to existing spring-mass models which have to be solved numerically and, as such, are prone to errors. Thus, here we present a two different application to generate generic motion for humans and fish locomotion. The resulting 
animation can be also transferred to existing mesh models of the same topology without losing the nature of the original animation of generated by the corresponding PDE geometry. Thus, the user has the possibility of choosing between the PDE-based animation or the one associated with a more complex geometric model depending on the specific end application and the computing resources available for that application.

This work is organised as follows. The rest of Section 1 provides a brief description on the theory asscociated with the solution of the PDEs in use throughout this work. Section 2 describes the methodology undertaken for both skeletal based animation and spine driven animation. Section 3 provides details of the process of retargeting the animation to a different mesh model with similar topology. Section 4 presents results and examples obtained for both approaches. Finally, Section 5 outlines the conclusions and future directions in which this work may be continued.

The rest of this work is organized as follows: Section 2 describes the modelling technique for producing cyclic motions of a human body and Section 3. Section 4 presents several examples of various cyclic motions and finally the conclusions of this work are presented in Section 5. components, incorporating the applicable criteria that follow.

\subsection{PDEs in Geometric Design}

Partial differential equations (PDEs) have been extensively used in computer graphics and computer aided design for generating and manipulating complex geometry. This kind of geometry is often referred as PDE surfaces and is a graphical representation of the solution to a given PDE. Among the advantages of using PDEs to generate surfaces over interpolation techniques such as splines are the significant reduction on the number of parameters required to generate a complex piece of geometry. This is because PDE based techniques utilises the notion of mathematical boundary-value problems. PDEs enable to unify of geometric and physical aspects of modelling.

Both, implicit and parametric geometry can be generated using PDEs. For instance, implicit PDE surfaces can be utilised for free-form surface construction [4] noise reduction [31] and image impainting [5]. They are usually the result from an evolution equation and are regarded as a collection of points satisfying a given geometric flow.

Parametric PDE geometry, on the other hand, results from the solution to an elliptic PDE in a parametric domain and represents a powerful geometry generation technique. Here the discretisation of the operator associated with the elliptic PDE represents an averaging process of the solution neighbourhood at the PDE and thus, automatically guarantees a certain degree of smoothness. The applications that have benefited from this comprise, surface blending [6], physics based models animation ( [25], [7]) and surface sculpting [8].

For the sake of brevity, the reader is referred to [10] for more details on the use of PDEs in geometric design. The mathematical foundations of the generalised elliptic PDE over a parametric domain are given below.

\subsubsection{Generalised elliptic PDEs}

Parametric surfaces generated through the use of elliptic PDEs are thought as the solution to such a PDE in two-dimensional parametric domain which is then mapped on to a three-dimensional space. The general form of an $2 N$-order elliptic PDE is given by,

$\left(\frac{\partial^{2}}{\partial u^{2}}+\frac{\partial^{2}}{\partial v^{2}}\right)^{N} \mathbf{X}(u, v)=0$

where $\mathbf{X}(u, v)$ represents the function giving rise to the parametric surface, $u$ and $v$ are the parametric surface coordinates (which are then mapped onto $(x(u, v), y(u, v), z(u, v))$

to generate the three-dimensional object), and $N \geq$ 1 determines the order of the PDE.

The general solution to Equation (1) can be written analytically, provided that the $2 N$ boundary conditions necessary to find a particular solution to this equation are periodic. Thus, the solution through separation of variables can be written as,

$\mathbf{X}(u, v)=\mathbf{A}_{\mathbf{0}}(u)+\sum_{n=1}^{\infty}\left[\mathbf{A}_{\mathbf{n}} \cos (n v)+\mathbf{B}_{\mathbf{n}} \sin (n v)\right]$,

where,

$$
\begin{aligned}
& \mathbf{A}_{\mathbf{0}}=\mathbf{a}_{\mathbf{0 0}}+\mathbf{a}_{\mathbf{0 1}} u+\mathbf{a}_{\mathbf{0 2}} u^{2}+\ldots+\mathbf{a}_{\mathbf{0}(\mathbf{2 N}-\mathbf{1})} u^{2 N-1}, \\
& \mathbf{A}_{\mathbf{n}}=\mathbf{a}_{\mathbf{n} 1} e^{a n u}+\mathbf{a}_{\mathbf{n} 2} u e^{a n u}+\mathbf{a}_{\mathbf{n} \mathbf{3}} e^{-a n u}+\mathbf{a}_{\mathbf{n} \mathbf{4}} u e^{-a n u} \\
& +\ldots+\mathbf{a}_{\mathbf{n}(\mathbf{2 N}-\mathbf{3})} u^{N-2} e^{a n u}+\mathbf{a}_{\mathbf{n}(\mathbf{2 N}-\mathbf{2})} u^{N-1} e^{a n u}, \\
& +\mathbf{a}_{\mathbf{n}(\mathbf{2 N}-\mathbf{1})} u^{N-2} e^{-a n u}+\mathbf{a}_{\mathbf{n}(\mathbf{2 N})} u^{N-1} e^{-a n u}, \\
& \mathbf{B}_{\mathbf{n}}=\mathbf{b}_{\mathbf{n} \mathbf{1}} e^{a n u}+\mathbf{b}_{\mathbf{n} \mathbf{2}} u e^{a n u}+\mathbf{b}_{\mathbf{n} \mathbf{3}} e^{-a n u}+\mathbf{b}_{\mathbf{n} \mathbf{4}} u e^{-a n u} \\
& +\ldots+\mathbf{b}_{\mathbf{n}(\mathbf{2 N}-\mathbf{3})} u^{N-2} e^{a n u}+\mathbf{b}_{\mathbf{n}(\mathbf{2 N}-\mathbf{2})} u^{N-1} e^{a n u} \text {, } \\
& +\mathbf{b}_{\mathbf{n}(\mathbf{2 N}-\mathbf{1})} u^{N-2} e^{-a n u}+\mathbf{b}_{\mathbf{n}(\mathbf{2 N})} u^{N-1} e^{-a n u},
\end{aligned}
$$

where the vector valued constants $a_{i j}$ and $b_{i j}$ with $\mathrm{i}=1, \ldots, \mathrm{n}$ and $j=1, \ldots, 2 N$ are determined by the specified boundary conditions. Given that the boundary conditions have been assumed to be periodic, they can be expressed in terms of a Fourier series. Let $\mathbf{C}^{\mathbf{j}}(v)$ represent the boundary conditions for which the solution to Equation general wants to be found, its Fourier series is then given by,

$$
\mathbf{C}^{\mathbf{j}}(v)=\mathbf{C}_{\mathbf{0}}^{\mathbf{j}}+\sum_{n=1}^{\infty}\left[\mathbf{C}_{\mathbf{n}}^{\mathbf{j}} \cos (n v)+\mathbf{S}_{\mathbf{n}}^{\mathbf{j}} \sin (n v)\right],
$$


reducing the problem to three linear systems of algebraic equations, which is approximated when the series is truncated to a finite value $M$, leading to,

$$
\begin{aligned}
\mathbf{X}(u, v) & =\mathbf{A}_{\mathbf{0}}(u)+\sum_{n=1}^{M}\left[\mathbf{A}_{\mathbf{n}} \cos (n v)+\mathbf{B}_{\mathbf{n}} \sin (n v)\right] \\
& +\mathbf{R}(u, v),
\end{aligned}
$$

where, $\mathbf{R}(u, v)$ is a function defined as,

$$
\begin{aligned}
\mathbf{R}(u, v) & =\mathbf{r}_{\mathbf{1}} e^{w u}+\mathbf{r}_{\mathbf{2}} u e^{w u}+\mathbf{r}_{\mathbf{3}} e^{-w u}+\mathbf{r}_{\mathbf{4}} u e^{-w u} \\
& +\ldots+\mathbf{r}_{\mathbf{2 N}-\mathbf{3}} u^{N-2} e^{w u}+\mathbf{r}_{\mathbf{2 N}-\mathbf{2}} u^{N-1} e^{w u} \\
& +\mathbf{r}_{\mathbf{2 N}-\mathbf{1}} u^{N-2} e^{-w u}+\mathbf{r}_{\mathbf{2} \mathbf{N}} u^{N-1} e^{-w u}
\end{aligned}
$$

where $w$ has been conveniently chosen as $w=a(M+1)$.

The vector-valued functions $\mathbf{r}_{\mathbf{j}}$, depend on $v$ and guarantee that the approximate solution satisfies the original boundary conditions. Each of the components of these vectors corresponds to a direction in the cartesian three-dimensional space.

Equation (7) is then composed mainly of two parts, one of them is composed of $A_{0}(u)$ fully determined in Equation (3), which is in fact a polynomial of order $2 N-1$ on $u$. The other part comprises the remaining terms, which depend on both $u$ and $v$.

The use of Equation (1) offers a simple yet effective method for characterizing the spine since it is fully determined in Equation (2). In crude terms, the spine of an object can be thought as the skeleton or backbone of the surface. Thus, the term $\mathbf{A}_{\mathbf{0}}$ in Equation (2) represents the spine of the object that is being modelled.

The remaining terms in Equation (7) can be considered as the radial component of the surface since they determine the distance by which a given point in the surface is driven away from the spine.

It is important to stress that the use of Equation (1) as a surface generation technique is not restricted to periodic boundary conditions since its solution can still be found numerically. Additionally, a particular case Equation (1) is that when $N=2$, representing the Biharmonic equation, which is well known in a number of disciplines such as fluid and solid mechanics and thus its solution is fully obtained when prescribing four boundary conditions. Therefore, the order of the PDE responsible for generating the surface is not restricted. For instance, the works presented in [35] and [33] make use of a sixth order elliptic PDE to obtain a fast surface generation technique whilst guaranteeing a higher degree of continuity over the generated surface.

Figure 1 shows an example of a surface generated using the Biharmonic equation and its corresponding spine. This surface has been obtained using a mesh grid 21 by 21 points thus, the spine has been computed over 21 points.
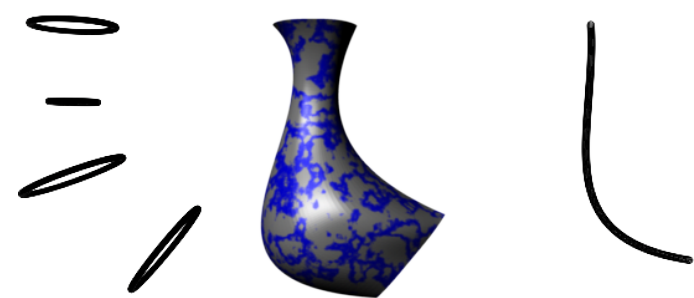

Fig. 1 Example of a PDE representation of a surface obtained by using the biharmonic equation. The spine of the surface is also outlined.

Previously, the spine has been used as an efficient method for parameterising complex surfaces as described in [28]. This work uses the spine of the object as an interactive tool for changing the shape of a given object in a fairly intuitive manner. Some other advantages offered by the use of this particular type of PDE surfaces are that this is a fast and accurate method for representing complex geometries, guarantees smoothness between surface patches, and the resolution with which the object is displayed can vary according to the specific needs of the user. Note that the method presented above is not restricted to model tubular shapes. Complex geometries are modelled by blending several surface patches. These patches are blended with the main body by extracting a suitable blending curve on the surface describing the length of the fish then, three additional curves are needed for each of the fins so that a PDE-based surface can be obtained $[29,30]$.

\section{Methodology}

The procedure for achieving both skeletal based animation of human motion and spine based animation of fish locomotion is divided into two stages: construction of PDE geometry and the corresponding animation. The first of these stages is carried out in a similar manner for both procedures. The second stage is the one characterizing each procedure. Moreover, spine based animation exploits the existence of analytic expression for the spine associated with the PDE surface as an animation engine and therefore, the properties of PDE based surfaces are exploited further. Details on both stages are given below.

\subsection{Construction of PDE geometry}

The process of constructing a suitable PDE-based representation of a model to be animated can be achieved by either creating a number of curves denoting the contour of the character within a CAD environment and 
then using them as boundary curves to generate the corresponding PDE surfaces or by extracting curves from an existing mesh model of the character and proceed accordingly.

The PDE surfaces employed in this work were obtained by extracting curves from an existing geometric model of a human body and some different types of fish so that they can be animated. These curves were extracted using Maya software and then exported to a $\mathrm{C}++$ environment to calculate the corresponding PDE geometry. These cross-sectional curves are essentially composed of vertices of the original mesh model so that the PDE-based surface preserves the realism of the mesh model as accurately as possible. It is worth mentioning that more than four curves have to be extracted from the geometric model in order to obtain a realistic PDE representation.

Thus, the PDE based geometry representing a human body is shown in Figure 2. The curves presented in Figure 2.a were extracted form an already existing mesh model and then a number of PDE surface patches were obtained. The resulting PDE based geometry is presented in Figure 2.b. It is important to stress that blending between the limbs and the torso has been by extracting the corresponding blending curve on the surface of the torso so that the limbs could be joined to it smoothly.

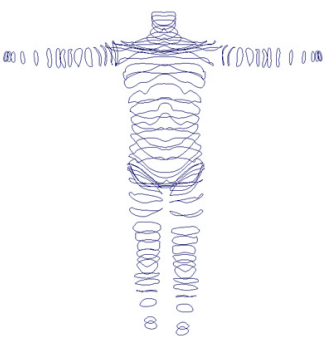

(a)

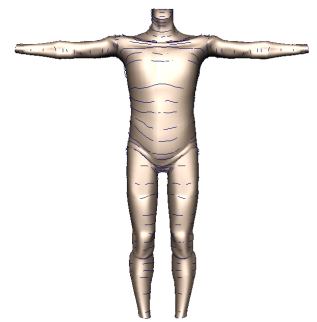

(b)
Fig. 2 PDE surface representing a human body: generating curves(a), resulting PDE surface(b).

Similarly, the PDE based geometry representing a dolphin was obtained. Figure 3 shows the original geometric model of a dolphin from which a set of 37 boundary curves has been extracted leading to its corresponding PDE surface. Additionally, the spine associated with this PDE surface representation is also outlined. The original geometric model is composed of 2932 vertices and the PDE configuration has been calculated over a grid of 41 by 41 points.

Once the PDE based representation of a human body and different types of fish (these are shown later)

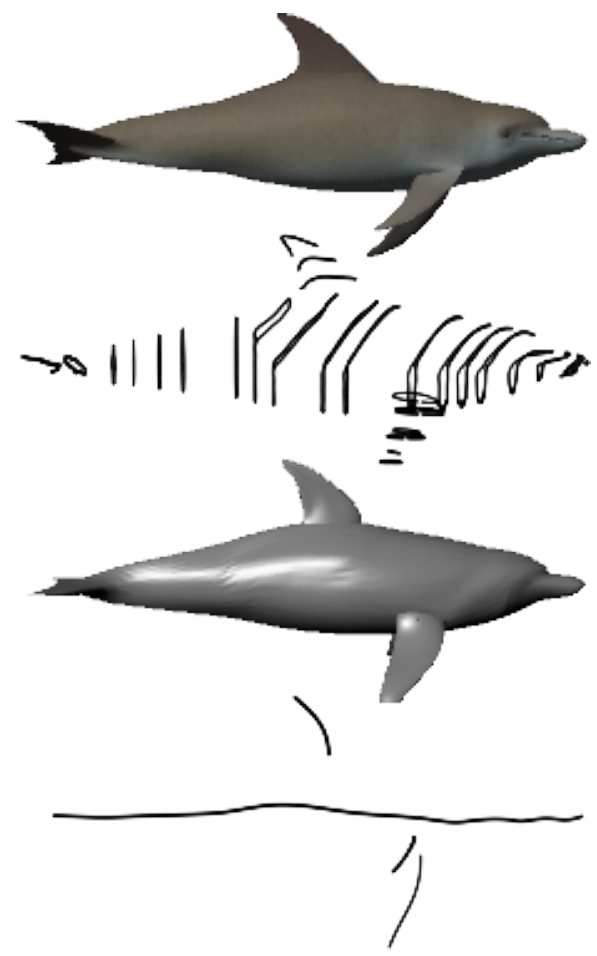

Fig. 3 Original geometric model representing a dolphin, the set of extracted curves from the original model together with the resulting PDE surface obtained using Equation (1) with $N=2$ and its corresponding spine.

has been obtained, details on how skeletal and spine based animations were linked to the use of PDE surface representations are given below.

\subsection{Skeletal based animation}

A skeletal system is composed of a set of bones or joints that define and control the movement [21,22]. These joints are hierarchically organized so that the influence of manipulating one of these joints has on the remaining ones is included. For the particular case of human animation, the skeleton system represents a simplified version of the skeleton of a human body. The skeletal system shown in Figure 4.a contains the joints and several controls for different parts of the body, each control can manipulate the part of the body it is connected with. Figure 4.b, shows the IK (inverse kinematics) joints and handles responsible for controlling the animation.

The creation of believable character motion requires controls based on how real bodies work [13]. For this purpose, the skeleton needs to be carefully bound with the model so that the skin determined by the PDE surface. This will blend with the animation as if it had a real skeleton inside it. 


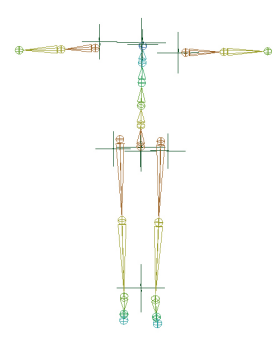

(a)

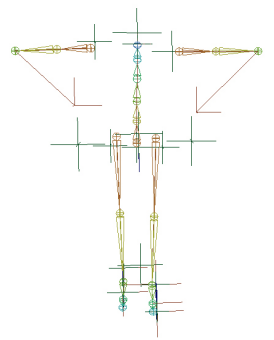

(b)
Fig. 4 Components of a skeletal system: joints(a), IK handles (b).

\subsubsection{Skinning}

Skinning s the process through which the surface representing a character is bound to the skeletal system [12]. The skin in this case consists of the PDE surface representation of a human body and needs to be bound to the skeletal system. To that end the boundary curves responsible for generating the PDE based surface representation of the human body are attached to the skeletal system. By skinning an object to a skeleton, the joint deformations are applied to the skin (surface) and thus deforming the surface itself. This represents a means for animating an object through the manipulation of the skeletal system. Note that the process of fitting the curves with the joints accurately requires further consideration [3] since problems may occur if the curves are not aligned properly with the joints and can result in unwanted wrapping effects. In this work, this problem has been addresses by carefully aligning the points representing each curve. Figure 5 shows a schematic representation of the skinning process. Here, the boundary curves responsible for generating the PDE have been bound to the skeletal system.

Once the curves have been attached to the skeletal system, it is necessary to create different poses so that the animation can be produced using interpolation technique between the poses. These poses can be found either manually or using mathematical expressions. To control the movement of each part of the body. This work makes use of a set of mathematical expression to create the poses.

\subsubsection{Mathematical expressions controlling the movement}

Mathematical expressions for defining the position of the joints in a skeletal for the animation of cyclic human motion can be found in [1] and, for the sake of brevity, the general idea behind the set of mathematical expression in the mentioned work is presented here.

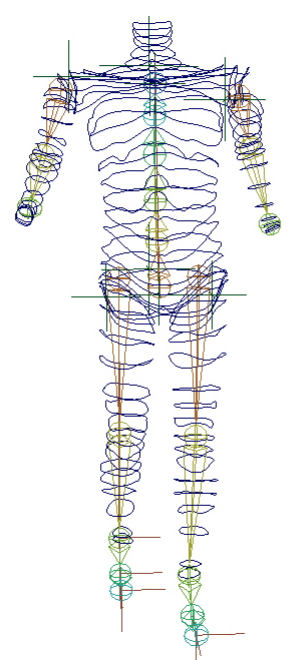

Fig. 5 Skinning process. The curves are attached to the skeletal system.

The general motion of body parts during cyclic motion is controlled by periodic functions such as sines and cosines. Let $x$ be a coordinate independent to the described movement, $y$ the coordinate describing the height of the human body and $z$ the direction of the movement itself. Thus, the translation associated with a given cyclic motion of a given body part is given by

$T_{y}=\sin (\omega t)$

$T_{z}=\cos (\omega t)$,

where $T_{y}$ and $T_{z}$ are functions representing the translation in $y$ and $z$ respectively, $t$ denotes time and $\omega$ the frequency of the movement. Note that since both directions are using the same frequency, their speed is also the same. Some adjustments to simple periodic function are required and each joint is identified over the system. For instance the equations modelling the animation of the right foot are given by

$R_{F_{y}}=T_{y}\left(H+H_{R}\right)+H_{R F}$
$R_{F_{z}}=-T_{z}\left(L+L_{R}\right)$

where $H$ controls the height of the step and is applied to both feet, $H_{R}$ and $H_{R F}$ are fine tune parameters controlling the step height of the right foot and the initial height of the right foot respectively. Note that these two parameters are generally set to zero by default. Similarly, $L$ controls the length of the step and is applied to both feet whilst $L_{R}$ is a fine tune parameter adjusting the length of the step given by the right foot (its default value is set to zero). The negative sign in the $z$ direction is to ensure that the feet are moving in 
opposite directions. Therefore the equations responsible for modelling the movement of the left foot do not include this negative sign.

Some of the poses composing the animation cycle associated with walking are presented in Figure 6 .

\subsection{Modelling fish locomotion through the PDE spine}

As far as spine based animation is concerned, once the PDE based geometry has been defined and the the spine of such geometry is identified, the next stage consists of finding a suitable mathematical relation for modelling the undulatory character of fish locomotion through the use of the spine. The mathematical function will be applied to the spine so that it facilitates the oscillating effect on the PDE surface. Moreover, it is desired that the analytic expression provides a certain degree of liberty to the user in the sense that adjusting the model to a different movement mode should not represent a burden to the user. For this purpose, different parameters, which can be adjusted at the user's discretion, are included in the analytic expression.

The analytic expression used to manipulate the spine, denoted by $S p_{i}(u, t)$, throughout an animation cycle is given by,

$S p_{i}(u, t)=S p_{\text {original }}+\Omega(u) \cos (\alpha u+\phi) \sin \left(2 \pi \omega t_{i}\right)$,

where $\Omega(u)$ determines the amplitude and depends upon $u, \phi$ represents the phase, $\alpha$ denotes the wave number and $\omega$ regulates the frequency of the undulatory movement. The subscript $i$ determines the frame for which the animation cycle, and $t_{i} \in[0,1]$ is the time associated with each respective frame. Notice that $u$ also requires to be normalised so that its value varies from 0 to $2 \pi$.

Equation (11) preserves the undulatory character inherent to the travelling wave formulation originally used to simulate fish locomotion and when assigning some particular values to the former set of parameters. i.e. Equation (11) represents the travelling wave formulation given in [16] by

$F(u, t)=A(u) \sin \left(\frac{2 \pi}{\lambda}(u-\omega t)\right)$,

where $A(u)$ is the amplitude, $\lambda$ is the wave number, $\omega=$ $2 \pi f$ is the pulsation ( $f$ representing the frequency) and $t$ denotes time.

This new formulation can be thought as a cyclic perturbation of the original position of the spine over a unit of time. Moreover, this equation can represent any of the four forms in which the movement observed in fishes has been classified.
It is important to stress that Equation (11) is responsible for the animation of the segment of the spine representing the length of the fish. The remaining segments of the spine, usually associated with dorsal and pectoral fins, experiment a displacement since the core of the surface has been modified the blending curve has been modified and therefore, the associated surface patches associated with these regions will require to be re-calculated on every frame.

Figure 7 illustrates how the spine is modified over one cycle of the undulatory movement. The horizontal line represents both the initial and final position of the spine, whereas the remaining outline its position at different values of $t$

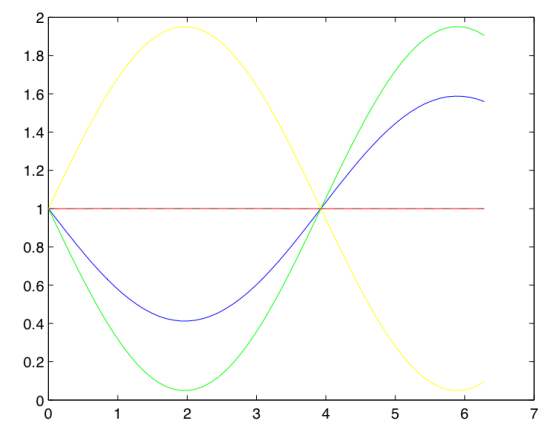

Fig. 7 Evolution of the spine over a cycle.

Animation of the PDE surface representation is then achieved by manipulating the spine associated with the model to be animated and afterwards adding to each point in the parametric domain its corresponding original radial component accordingly at any given frame.

It is clear that in this formulation conservation of volume is not guaranteed. However, it is not a cause for concern since a well chosen set of parameters would maintain the change of volume within reasonable limits whilst not compromising the realism of the model and thus the visual effect of this change of volume would not be visible to the eye.

Figure 8 shows the original PDE surface representation of the fish shown in Figure 3 and two different frames of the animation cycle. The spine associated with each of these PDE surfaces has also been outlined in the figure.

Once an animation cycle consisting of $N$ frames has been obtained over the PDE surface representation, the animation can be transferred to either the original mesh model from where the boundary curves associated with the PDE surface were extracted or to another mesh model with the same topology. 

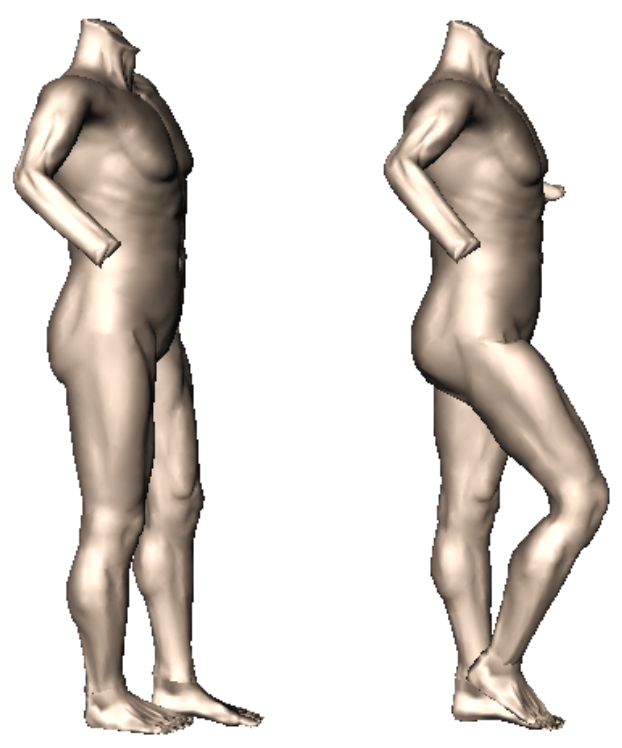

Fig. 6 Different stages of an animation cycle for walking.
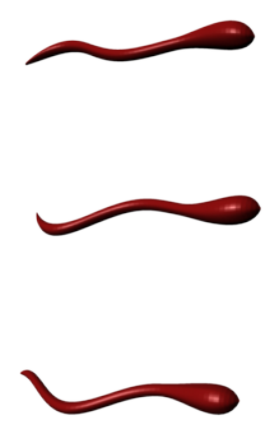

Fig. 8 PDE surface representations of an eel at different times over an animation cycle. The starting configuration of the eel and two different frames are portrayed here. The spines associated with these surfaces are also shown.

\subsection{Motion re-targetting}

Motion retargeting techniques consists of simply transferring existing motions to different target objects in order to achieve the desired effect. Given that motion is difficult to generate from scratch using traditional methods for different characters, the use of existing motions is a faster alternative to obtain the animation $[9,11]$. The process of transferring the animation from a PDE surface representation to any mesh model representing the same type of fish is carried out as follows:

- Alignment of the initial PDE surface representation and the target mesh model in the same initial position, that is, key features of the models have to be
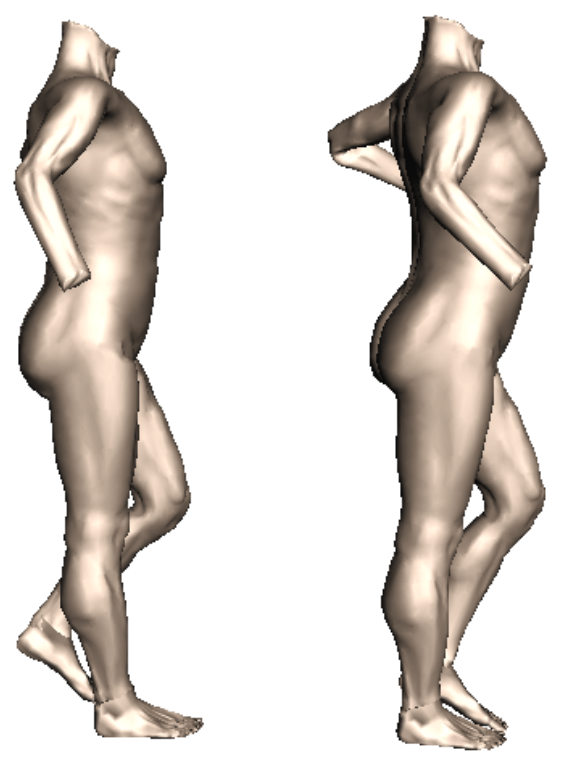

positioned so that they nearly overlap. This facilitates the mapping correspondence between the two surfaces.

- Mapping correspondence between models. This process consists of associating each point of the mesh model with the nearest point of the PDE surface representation in their initial configurations. Thus, each point in the mesh model is represented on the PDE surface by a given point in the PDE surface and it is assumed that this point remain as the closest one to the same particular point at any frame throughout the animation cycle.

- Animation of the mesh model is carried out by finding the difference between the resulting PDE surface at a given frame and the original one for each point. Then, this difference is added to each point of the original mesh model according to the mapping correspondence previously found. The procedure is repeated for each frame of the animation cycle.

The advantage of using motion re-targetting when using PDE based surface representation is that two different representations of the same object can be animated simultaneously. This provides the user the opportunity to choose between a complex or a simplified representation, depending on storage and memory resources associated with the end application where the animation is required. Note that the quality of the mapping correspondence between the PDE-based representation and the original mesh model depends on the resolution of the grid used to compute the PDE-based rep- 
resentation. Thus, using a grid with a similar number of points to the number of points in the original mesh will produce an acceptable mapping correspondence.

Figure 9 shows the initial and two additional frames of the animation cycle for both the PDE surface representation and the mesh model from where the boundary curves were extracted. The animation was transferred according to the procedure described above.

\section{Results and examples}

In the interest of illustrating how to obtain different types of human cyclic motion from the skeletal based platform developed here, Different types of movement were modelled. One of the movements that was assessed was that of running. Figure 10 shows different stages of a running cycle.

Additionally, the technique proposed here for motionretargetting was employed to transfer different animation sequences from the initial PDE based geometry to different mesh models with similar topology. Figure 11 shows some examples on how the animation was transferred from a PDE surface representation of a human to a mesh model of a man wearing a suit. The PDE based representations are shown in the left hand side of the figure.
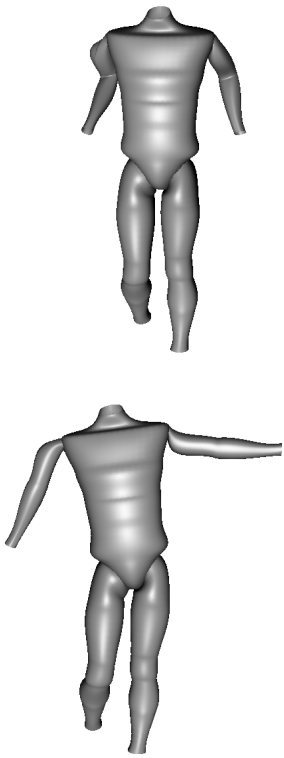

(a)
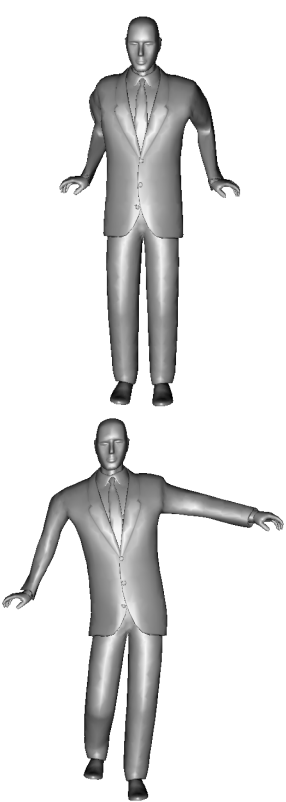

(b)
Fig. 11 Motion re-targetting from a PDE based representation to a mesh model with similar topology.
As far as fish locomotion is concerned and given that the undulatory movement of fish is mainly divided into four major types, at least one fish representing each of these categories has been chosen to be modelled and animated in this work. The fish representing the anguilliform movement is an eel. Fish swimming in subcarangiform are represented by a tropical fish. The trout has been selected as an suitable example of carangiform mode and the dolphin represents a fish thunniform swimming mode.

Mesh models of these fish have been found in [17]. Then, a set of boundary curves have been extracted from each model in order to obtain its respective PDE surface representation. Figure 12 presents the original mesh model, the set of extracted boundary curves and its corresponding PDE surface. The fish selected to represent this process has been the trout.
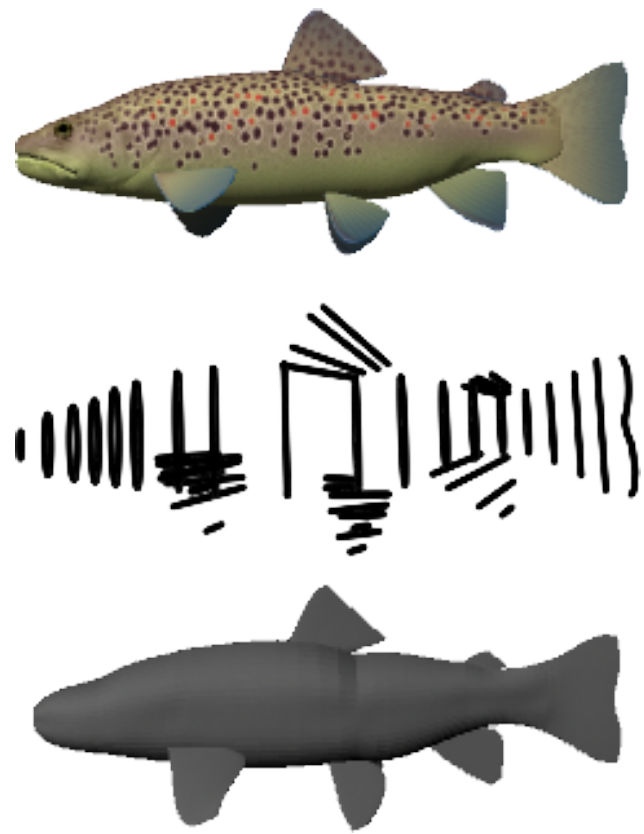

Fig. 12 Original mesh model of a trout, extracted boundary curves and resulting PDE surface representation for a trout.

Once the PDE surface representation of the models has been found, the spine has been manipulated according to Equation (11) and for the purpose of modelling each category accurately, the parameters in this equation have been adjusted so that the model is animated realistically. Table 3 lists the values of all the required parameters to manipulate the spine according to each of the fishes modelled in this work. The parameters are: amplitude $\Omega(u)$, wave number $\alpha$ and phase $\phi$.

Thus, the animation of the PDE surface representation of each fish is obtained over a cycle. The duration of the cycle has been set equal to one second and twenty- 

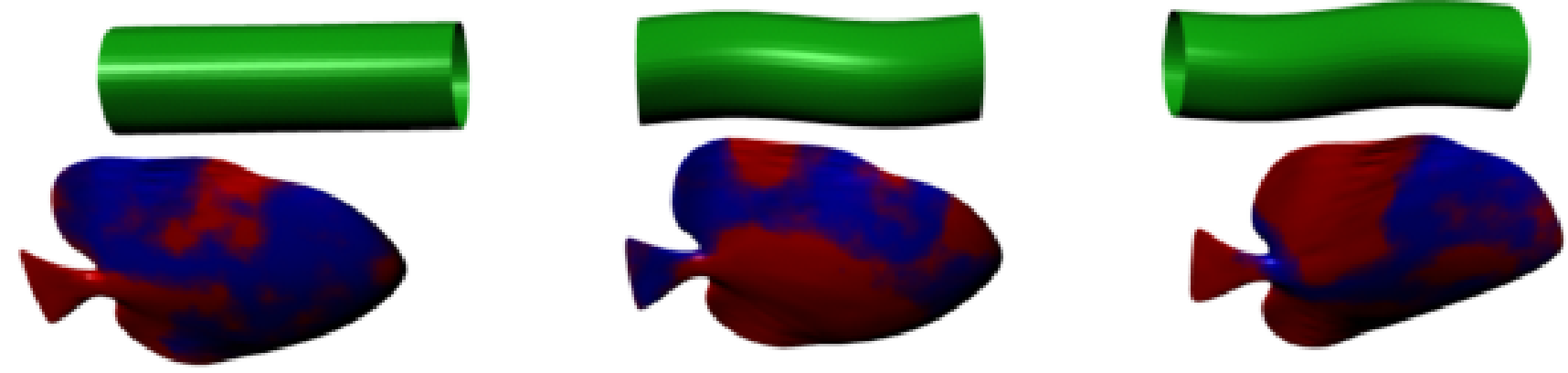

Fig. 9 PDE surface representation of cylinder and mesh model of the same fish at different times over an animation cycle. The initial configuration of the fish and two different frames are portrayed here for both the PDE surface and the mesh model.
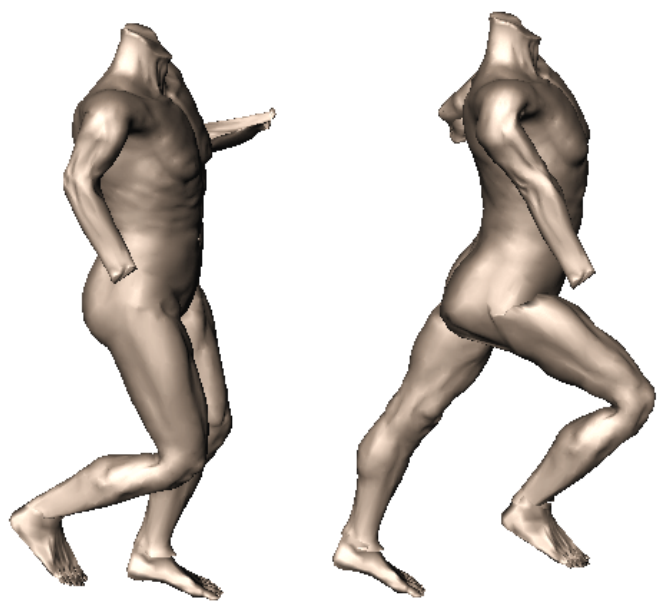

Fig. 10 Different stages of an animation cycle for runnining.

\begin{tabular}{llllll}
\hline \hline Movement & Fish & $\Omega(u)$ & $\alpha$ & $\phi$ & $\omega$ \\
\hline Anguilliform & Eel & $\frac{0.3 u}{2 \pi}$ & 1.5 & $\frac{\pi}{2}$ & 1.0 \\
Subcarangiform & Trout & $\frac{u}{2 \pi}$ & 0.75 & $\frac{\pi}{2}$ & 2.0 \\
Carangiform & Tropical fish & 0.1 & 1.0 & $\frac{\pi}{2}$ & 2.0 \\
Thunniform & Shark & $\frac{u}{2 \pi}$ & 0.25 & 0.0 & 1.0 \\
Thunniform & Dolphin & 1.0 & 0.75 & $\frac{\pi}{2}$ & 1.3 \\
\hline
\end{tabular}

Table 1 Parameters employed to manipulate the spine of a PDE surface representing each fish according to its corresponding type of swimming movement.

four frames have been obtained during each cycle. The results obtained after animating the PDE surface representations of an eel are shown in Figure 13 the first, sixth, twelfth, eighteenth and last frames are displayed for these fish.

Then, the animation was transferred to the original mesh model representing each fish from which the boundary curves were extracted by using a mapping correspondence between the PDE surface representation and the original mesh model as described before.
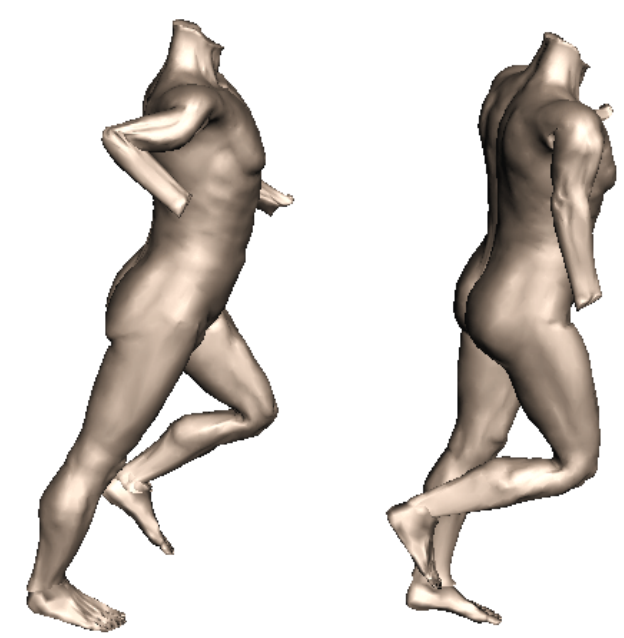

Figure 14 and Figure 15 includes frames extracted from the animation sequence after it was transferred to the original mesh model. The frame sequence presented in this figure is the same as the one presented in Figure 13. However, for the sake of brevity and completeness, the mesh models representing different types of fish are used. Thus, the animation of a mesh model representing a shark is shown in Figure 14, whereas a tropical fish, a trout and a dolphin are shown in Figure 15 .

It is important to stress that the number of frames can vary according to the specific needs of the particular application and can be computed easily thanks to the analytic nature of the mechanism driving the animation.

A video of these animations sequences has been included so that the reader can appreciate the full animation here for the models presented in this work using both PDE surfaces and mesh-based representations. Figure 16 shows some screenshots of such a video showing different types of fish simultaneously. 

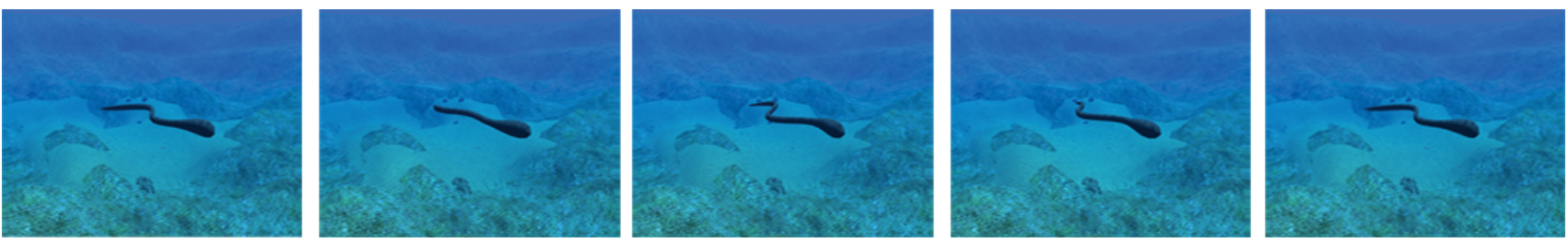

Fig. 13 Sequence of frames belonging to an animation cycle of PDE surface representation of an eel. The first, the sixth, twelfth, eighteen and last frame are shown.
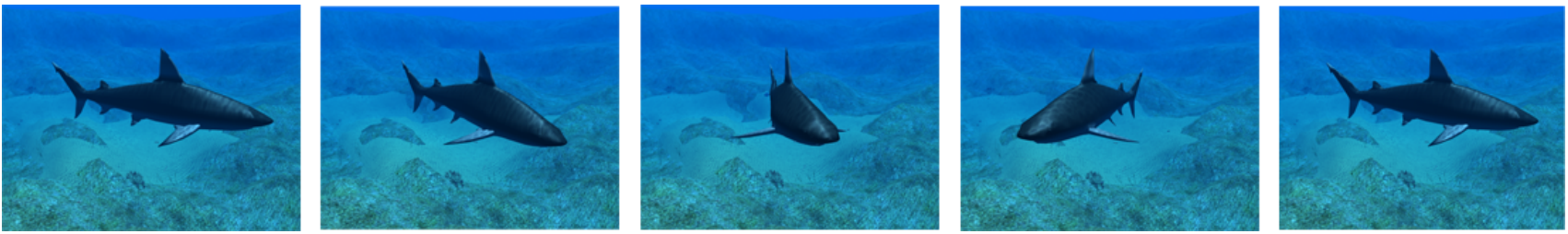

Fig. 14 Sequence of frames belonging to an animation cycle of a mesh model representing a shark. The first, the sixth, twelfth, eighteen and last frame are shown.
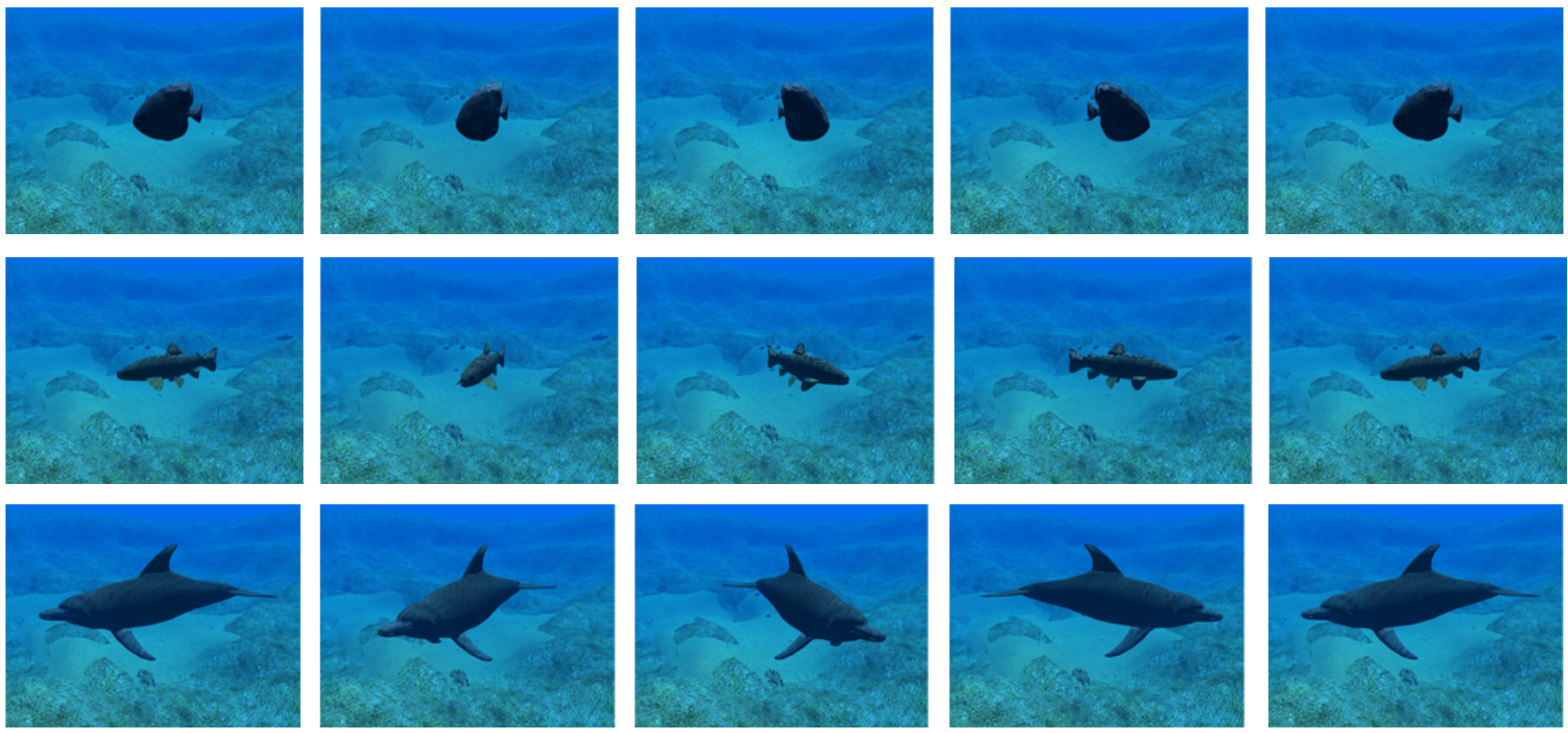

Fig. 15 Sequence of frames belonging to an animation cycle of the original mesh models associated with different fishes. The first, the sixth, twelfth, eighteen and last frame are shown for a tropical fish, a trout and a dolphin on each respective case.

Note that the characteristic undulatory movement observed in fish when swimming has been modelled in this work and thus, the reader may have the impression that the fish is swimming in still water or in vacuum. At present, the hydrodynamic effects provoked by the surroundings, such as currents and hydrodynamic drag have not been considered in this work; however, these effects can be considered as future directions for continuing this work. This can be carried out by coupling the relevant partial differential equations modelling the surrounding hydrodynamics by either including them directly into the PDE modeling the surface or by previously adjusting the generating curves. Moreover, The change in the direction in which fish swim is thought to be due to abrupt movements of the lateral fins. The modeling of such movements is represents a natural choice for extending this work and has been left for the future.

\section{Conclusions}

This work presents a technique for animating cyclic motions using partial differential equations, since these are powerful means for parameterizing complex surfaces in terms of a small set of design parameters. This is studied under two frameworks: human motion and fish locomotion. The first of these frameworks is carried out by 

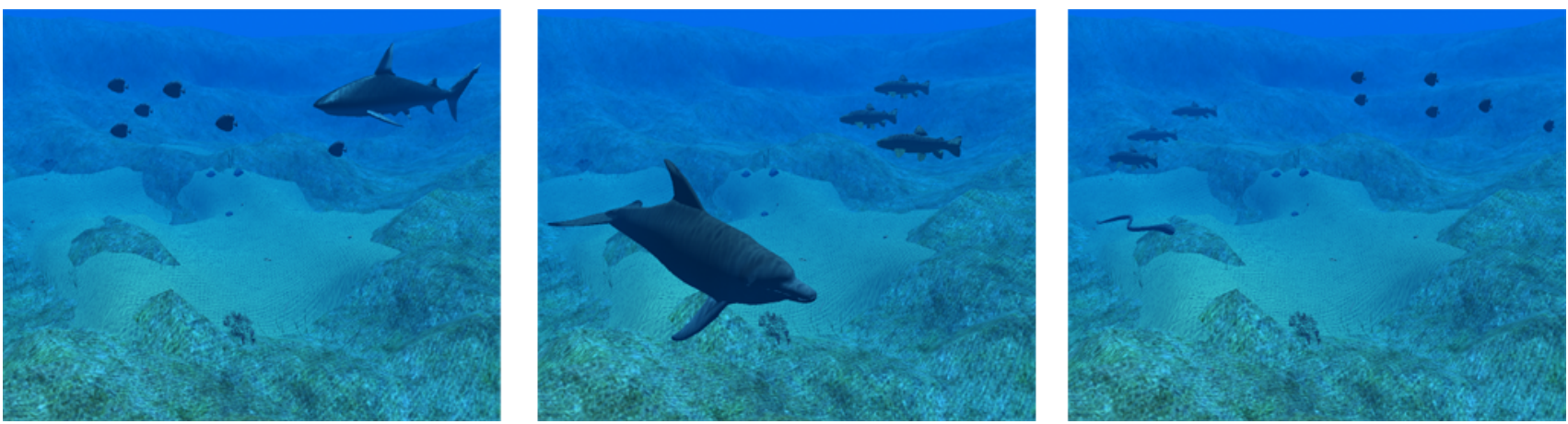

Fig. 16 Sequence of frames belonging to an animation cycle of a video showing different types of fish.

using skeletal based animation whereas the second one uses the spine of a PDE as an animation tool. The PDE surface for both frameworks is found using the general elliptic equation.

The boundary curves responsible for describing the geometry were attached to a skeletal system resembling the human skeleton and then, analytic expressions were used to manipulate the joints in the skeletal system. By contrast, spine based animation has been achieved by manipulating the segment of the spine associated with the backbone of the fish analytically. The analytic expression used to characterize the oscillatory nature of the motion observed in fish when swimming has been given in terms of a set of parameters that can be varied according to the type of propulsion of a given fish, leading to realistic animations. The technique proposed here represents a more reliable alternative than the already existing spring-mass model since it is faster and less prone to numerical errors.

The last stage of both frameworks consisted on transferring the animation from the PDE based geometry to different mesh models with similar topologies so that the user could have the ability to choose between the different models available representing the same geometry according to the storage and memory resources of the end application.

Future work includes the use of the spine for human cyclic animation to control the movement without the need of the skeletal system. Some other future directions for this work comprises the implementation of virtual environments as considered in [14] and the introductionof optimization techniques for the purpose of controlling the volume of the surface at each frame of the animation sequence.

\section{References}

1. H. Alaskari. Cyclic animation user interface in maya, 2005.
2. G. Allard. Control of a free-swimming fish using fuzzy logic. The International Journal of Virtual reality, 6(3):2328, 2003.

3. I. Baran and J. Popović. Automatic rigging and animation of 3d characters. ACM Trans. Graph., 26(3):72, 2007.

4. M. Bertalmio, L. Cheng, S. Osher, and S. Sapiro. Variational Problems and Partial Differential Equations on Implicit Surfaces. J. Comput. Phys., 174(2):759-780, 2001.

5. M. Bertalmio, G. Sapiro, V. Caselles, and C. Ballester. Image Inpainting. pages 417-424, New Orleans, USA, 2000. SIGGRAPH 2000.

6. M. I. G. Bloor and M. J. Wilson. Generating Blend Surfaces using Partial Differential Equations. Computer Aided Design, 21(3):165-171, 1989.

7. H. Du and H. Qin. Dynamic PDE surfaces with flexible and general geometric constraints. pages 213-222, Hong Kong, 2000b. Pacific Graphics 2000.

8. H. Du and H. Qin. Dynamic PDE-based surface design using geometric and physical constraints. Graphical Models, 67:43$71,2005$.

9. M. Gleicher. Retargetting motion to new characters. In $S I G-$ GRAPH '98: Proceedings of the 25th annual conference on Computer graphics and interactive techniques, pages 33-42, New York, NY, USA, 1998. ACM.

10. G. González Castro, H. Ugail, P. Willis, and I. J. Palmer. A survey on partial differential equations in geometric design. Visual Computer, 24:213-225, 2008.

11. C. Hecker, B. Raabe, R. W. Enslow, J. DeWeese, J. Maynard, and K. van Prooijen. Real-time motion retargeting to highly varied user-created morphologies. In SIGGRAPH '08: ACM SIGGRAPH 2008 papers, pages 1-11, New York, NY, USA, 2008. ACM.

12. D. L. James and C. D. Twigg. Skinning mesh animations. In SIGGRAPH '05: ACM SIGGRAPH 2005 Papers, pages 399-407, New York, NY, USA, 2005. ACM.

13. P. Kalra, N. Magnenat-Thalmann, L. Moccozet, G. Sannier, A. Aubel, and D. Thalmann. Real-time animation of realistic virtual humans. IEEE Comput. Graph. Appl., 18(5):42-56, 1998.

14. H. Lee, E. Kim, N. Joo, and G. Hur. Development of real time virtual aquarium system. IJCSNS International Journal of Computer Science and Network Security, 6(7):58-63, 2006.

15. L. Leronutti and L. Chittaro. Employing virtual humans for education and training in $\mathrm{x} 3 \mathrm{~d} / \mathrm{vrml}$ worlds. Comput. Educ., 49(1):93-109, 2007.

16. M. J. Lighthill. Hydromechanics of aquatic animal propulsion. Annual Review of Fluid Mechanics, 1(1):413-446, 1969.

17. Models. http://toucan.web.infoseek.co.jp, 2008.

18. F. Multon, L. France, M. P. Cani-Gascuel, and G. Debunne. Computer animation of human walking: a survey. The Jour- 
nal of Visualization and Computer Animation, 10(1):39-54, 1999.

19. D. Ormoneit, M. J. Black, T. Hastie, and H. Kjellstrom. Representing cyclic human motion using functional analysis. IVC, 23(14):1264-1276, 2005.

20. D. Ormoneit, M. J. Black, H. Sidenbladh, and T. Hastie. Learning and tracking cyclic human motion. Technical report, In A. Kent \& C.M. Hall (Eds.), Encyclopedia of Library and Information Science, 2001.

21. S. II Park and J. K. Hodgins. Capturing and animating skin deformation in human motion. ACM Trans. Graph., 25(3):881-889, 2006.

22. M. Pratscher, P. Coleman, J. Laszlo, and K. Singh. Outsidein anatomy based character rigging. In $S C A$ '05: Proceedings of the 2005 ACM SIGGRAPH/Eurographics symposium on Computer animation, pages 329-338, New York, NY, USA, 2005. ACM.

23. K. Stephens, B. Pham, and A. Wardhani. Modelling fish behaviour. In GRAPHITE '03: Proceedings of the 1st international conference on Computer graphics and interactive techniques in Australasia and South East Asia, pages 71-78, 2003.

24. D. Terzopoulos. Artificial life for computer graphics. Commun. ACM, 42(8):32-42, 1999.

25. D. Terzopoulos, J. Platt, A. Barr, and K. Fleischer. Elastically Deformable Models. Computer Graphics, 21:205-214, 1987.

26. D. Tost and X.Pueyo. Human body animation: a survey. The Visual Computer, 3(5):254-264, 1988.

27. X. Tu and D. Terzopoulos. Artificial fishes: physics, locomotion, perception, behavior. In SIGGRAPH '94: Proceedings of the 21st annual conference on Computer graphics and interactive techniques, pages 43-50. ACM, 1994.

28. H. Ugail. On the Spine of a PDE Surface. In M. J. Wilson and R. R. Martin, editors, Mathematics of Surfaces, pages 366- 376. Springer, 2003.

29. H. Ugail, M. I. G. Bloor, and M. J. Wilson. Manipulation of pde surfaces using an interactively defined parameterisation. Computers \& Graphics, 23(4):525-534, 1999.

30. H. Ugail, M. I. G. Bloor, and M. J. Wilson. Techniques for Interactive Design using the PDE Method. ACM Transactions on Graphics, 18(2):195-212, 1999.

31. G. Xu, Q. Pan, and C. L. Bajaj. Discrete Surface Modelling Using Partial Differential Equations. Computer Aided Geometric Design, 23:125-145, 2006.

32. X. Yang, D.C. Petriu, T.E. Whalen, and E.M. Petriu. Hierarchical animation control of avatars in 3d virtual environments. IEEE Transaction on Instrumentations and Measurements, 54(3):1333-1341, 2005.

33. L. You, P. Comninos, and J. J. Zhang. PDE Blending Surfaces with C2 Continuity. Computers and Graphics, 28(6):895-906, 2004.

34. Q. Yu and D. Terzopoulos. Synthetic motion capture: Implementing an interactive virtual marine world. The Visual Computer, 15(7/8):377-394, 1999.

35. J. J. Zhang and L. You. Fast Surface Modelling using a 6th Order PDE. Computer Graphics Forum, 23(3):311-320, 2004.

36. Q Zhu, M. J. Wolfgang, D. K. P. Yue, and M. S. Triantafyllou. Three-dimensional flow structures and vorticity control in fish-like swimming. Journal of Fluid Mechanics, 468:1-28, 2002 . 